\title{
Gel immersion endoscopy: Innovation in securing the visual field - Clinical experience with 265 consecutive procedures $\square$
}

\section{다)(요 $\odot$}

\author{
Authors \\ Yamamoto ${ }^{1}$ \\ Institutions \\ 1 Division of Gastroenterology, Department of Medicine, \\ jichi Medical University, Shimotsuke, Tochigi, Japan \\ 2 Shinozaki Medical Clinic, Utsunomiya, Tochigi, Japan \\ 3 Department of Surgery, Jichi Medical University, \\ Shimotsuke, Tochigi, Japan
}

Tomonori Yano', Takahito Takezawa', Kousei Hashimoto', Ayako Ohmori', Satoshi Shinozaki², Manabu Nagayama', Hirotsugu Sakamoto', Yoshimasa Miura'1, Yoshikazu Hayashi ${ }^{1}$, Keijiro Sunada', Alan Kawarai Lefor ${ }^{3}$, Hironori

submitted 12.10 .2020

accepted after revision 15.2.2021

Bibliography

Endosc Int Open 2021; 09: E1123-E1127

DOI 10.1055/a-1400-8289

ISSN 2364-3722

\author{
(C) 2021. The Author(s). \\ This is an open access article published by Thieme under the terms of the Creative \\ Commons Attribution-NonDerivative-NonCommercial License, permitting copying \\ and reproduction so long as the original work is given appropriate credit. Contents \\ may not be used for commercial purposes, or adapted, remixed, transformed or \\ built upon. (https://creativecommons.org/licenses/by-nc-nd/4.0/) \\ Georg Thieme Verlag KG, Rüdigerstraße 14, \\ 70469 Stuttgart, Germany
}

Corresponding author

Tomonori Yano, Jichi medical University - Department of Medicine, Division of Gastroenterology, 3311-1 Yakushiji

Shimotsuke, Tochigi 329-0498, Japan

Fax: +81-285-44-0047

tomonori@jichi.ac.jp

\section{Introduction}

During endoscopy, especially in patients with gastrointestinal bleeding, it is often difficult to secure the visual field. Although water immersion techniques are sometimes used to mitigate this situation [1-3], maintenance of the visual field is often difficult because the infused/injected water rapidly mixes with any luminal blood and/or residue. Poor preparation before colonoscopy also makes it difficult to secure the visual field when using water immersion or water exchange techniques. The use of gas insufflation to displace luminal contents can cause significant discomfort and difficult endoscope insertion. It is also often impossible to adequately aspirate blood, clots and residue. Although positional changes may improve visualization through gravity, this strategy is also ineffective at times.

To solve these problems, we developed an innovative technique called "gel immersion endoscopy" [4]. Clear gel with an appropriate viscosity (which prevents rapid luminal mixing) is injected/infused through the accessory channel, instead of water. The viscous gel displaces luminal blood, clots and residue ( $\triangleright$ Fig.1). In the space occupied by the gel, we can then easily secure the visual field and perform endotherapy calmly and effectively, even in patients with massive arterial bleeding and/or poor preparation ( $\triangleright$ Video 1 ).
We use a jelly-like drink "OS-1 jelly" (Otsuka Pharmaceutical Factory, Tokushima, Japan) for this technique. This is a dedicated rehydration-supplement for patients with dehydration which consists of a gelling agent (polysaccharide-thickener), electrolytes, carbohydrates, and water. Since it is a food-supplement, it is considered safe for use within the digestive tract.

This technique was reported with small number of procedures [4-7]. However, the effectiveness and safety of this technique has not yet been studied in a large number of procedures. The aim of this study is to clarify the effectiveness and safety of the gel immersion endoscopy technique for the management of gastrointestinal bleeding and/or endoscopy after poor preparation.

\section{Patients and methods}

From June 2012 until December 2017, 265 consecutive procedures using gel immersion endoscopy (median age 67 years, age range 1 to 94, male/female: 167/98) for 222 patients were identified by searching the electronic medical records. These procedures include 93 esophagogastroduodenoscopies, 108 colonoscopies, 45 anterograde double-balloon enteroscopies, 14 retrograde double-balloon enteroscopies and five other procedures. Gel immersion endoscopy was used for bleeding in 193 , for bleeding and poor preparation in 36 , for poor prepara- 


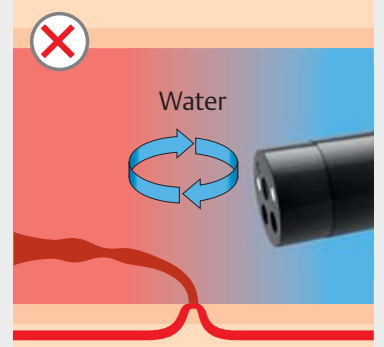

Water immersion endoscopy

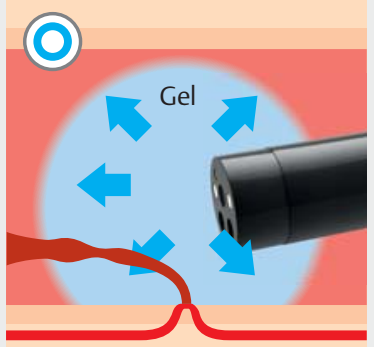

Gel immersion endoscopy
Fig. 1 Any infused/injected water rapidly mixes with any luminal blood and/or residue. But the viscous gel displaces luminal blood, clots and residue. Consequently, gel immersion endoscopy clarifies a bleeding source.

tion in 29, and for other reasons in seven. These 265 procedures were retrospectively evaluated. This was a retrospective study with opt-out consent and approved by the Institutional Review Board of Jichi Medical University. Only anonymized images and data were extracted from electronic medical records and analyzed. Of 265 procedures in this study, data from 17 procedures have been already published in the previous report [4].

The aim of procedures in patients with bleeding included both identifying the hemorrhagic lesion and confirming no hemorrhagic lesion in the observation range. The aim of procedures in patients with poor bowel preparation included both inserting the scope to the destination for subsequent procedures and observing the lesion for diagnosis. The aim of procedures in other patients included observing the postoperative intestine at low internal pressure, biliary cannulation through a choledochojejunostomy near the oozing neoplastic lesion, and keeping the injected contrast medium in the bile duct. Medical records including endoscopic images and unedited videos if available were independently evaluated by three gastroenterologists (TT, KH, AO). The evaluators judged whether the visual field was secured, not secured, or undecided. When the evaluator judged this method effectively secured the visual field to

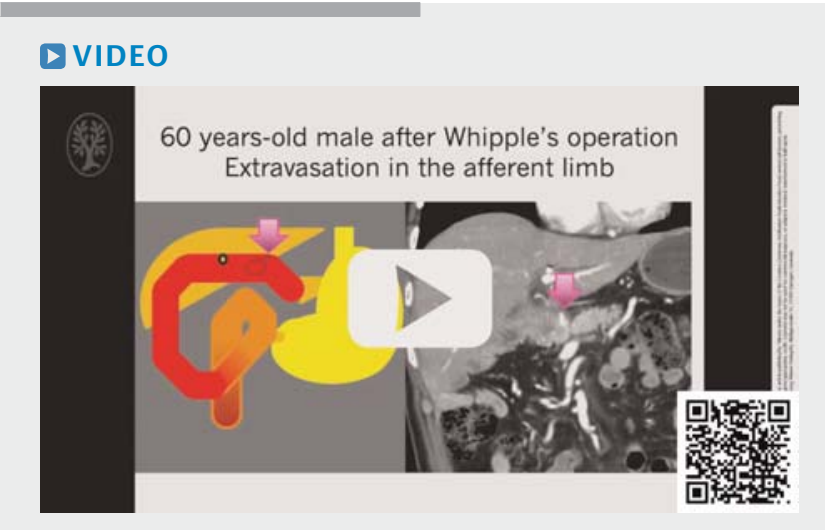

- Video 1 Two patients with gastrointestinal bleeding treated by using gel immersion endoscopy. achieve the aim of the procedure, it was defined as "secured." The final classification of each procedure was decided by majority vote. In the same way, procedure-related adverse events (AEs) and incidents were evaluated and a judgment was made as to whether the event could or could not have been prevented. The definition and severity grading system of AEs and incidents by the American Society of Gastrointestinal Endoscopy [8] was used.

\section{Results}

Of 265 total procedures, the visual field was secured/not secured/undecided in 233/21/11 ( $\triangleright$ Table 1 ). Comparing the success rates (secured/total) for securing the visual field in each organ, success rates were significantly higher in the large bowel only when comparing the stomach (35/44) and large bowel $(106 / 115)(P=0.025$, Chi square test).

The success rate in the second half of the study period (121/ $132)$ tended to be higher than in the first half $(112 / 133)(P=$ 0.063 , Chi square test).

Of 265 procedures, procedure-related incidents occurred in three and a severe AE occurred in one as described below.

Post-procedure abdominal pain was observed in two patients. One was an 84-year-old woman who underwent emergency anterograde double-balloon enteroscopy for diverticular bleeding in the ascending portion of the duodenum, which was controlled with endoclips. The other patient was a 66-year-old woman who underwent colonoscopy for bowel obstruction of the sigmoid colon due to malignant lymphoma which included obtaining a biopsy and performing a contrast study. In both patients, symptoms improved with non-operative management and their hospital stays were not prolonged.

Weight gain of $2.2 \mathrm{~kg}$ was observed in a 77 -year-old man with chronic renal failure on hemodialysis that occurred after anterograde double-balloon enteroscopy for jejunal bleeding from a Dieulafoy's lesion detected by using gel after failure to identify the lesion with water immersion and controlled with endoclips. Although his weight was adjusted by hemodialysis, the hospital stay was not prolonged.

Extension of mediastinitis was observed in one patient, a 79year-old woman with spontaneous esophageal rupture. The patient was admitted to an outside facility with abdominal pain and vomiting and diagnosed with a large sliding hernia by computed tomography (CT) scan. She was then transferred to our institution because of hematemesis. Urgent esophagogastroduodenoscopy revealed a very deep ulcer in the esophagus, which suggested spontaneous esophageal rupture. The esophageal rupture resulting in mediastinitis with an upside-down stomach was retrospectively confirmed by CT scan, which had been performed at the outside facility. Because the CT scan performed after esophagogastroduodenoscopy revealed extension of mediastinitis, she was treated surgically and discharged 3 weeks later. 
- Table 1 Success rate of securing the visual field according to organ.

\begin{tabular}{|l|c|c|c|c|c|}
\hline & Total & Secured & Not secured & Undecided & $\begin{array}{l}\text { Secured rate (\%) } \\
\text { (secured/total) }\end{array}$ \\
\hline Esophagus & 13 & 11 & 2 & 0 & 85 \\
\hline Stomach & 44 & 35 & 5 & 4 & $80^{*}$ \\
\hline Duodenum & 43 & 37 & 5 & 1 & 86 \\
\hline Jejunum & 27 & 23 & 1 & 3 & 100 \\
\hline lleum & 10 & 10 & 0 & 0 & $92.2^{*}$ \\
\hline Large bowel & 115 & 106 & 7 & 2 & 83 \\
\hline Afferent limb & 12 & 10 & 1 & 1 & 100 \\
\hline Bile duct & 1 & 1 & 0 & 0 & 87.9 \\
\hline Total & 265 & 233 & 21 & 11 & 85 \\
\hline * Success rate was significantly higher in the large bowel only when comparing the stomach and large bowel (P=0.025, Chi square test).
\end{tabular}

\section{Discussion}

This retrospective study showed that gel immersion endoscopy enables securing the visual field throughout the gastrointestinal tract. Due to the wide lumen, the success rate for securing the visual field in the stomach was significantly lower than inr the large bowel. Theoretically, gel immersion endoscopy is more effective with a narrow lumen, because a narrow lumen is often filled with blood, but easily replaced with gel. Although the success rate was satisfactory from the beginning of the study period, the success rate improved in the second half of the study as we gained more experience and understood when, where, and how to use the technique effectively.

In practical use, a large accessory channel with a BioShield irrigator (US Endoscopy (STERIS), Ohio, United States) enables additional injection of gel with a therapeutic device in situ within the accessory channel ( $\nabla$ Fig. 2 ). The use of a distal attachment makes it easier to concentrate the gel in front of the endoscope ( $\triangleright$ Fig. $\mathbf{3}$ ). Gel immersion endoscopy is also very well suited to use with a dedicated double-balloon endoscope (with or without the over-tube), even within the colon and esophagus. Insufflation of the endoscope-balloon can stop the backflow of gel, making it easier to push away blood, clots, and residue ( $\triangleright$ Fig.4). To fill the lumen with gel, the gel should be injected/infused after aspiration of any luminal gas (similar to the technique used for water immersion during endoscopic ultrasound). After injection of the gel, gas insufflation and suction should be avoided until hemostasis is achieved. This is because gas insufflation generates bubbles within the gel and suction causes blood, clots, and residue to once again displace the gel and obscure the view. The gel should be injected near the wall below any blood clots, especially in the stomach, since the bleeding point originates from vessels within the wall and not the center of the lumen ( $\triangleright$ Fig.5). An initial injection of around 50 to $100 \mathrm{~mL}$ of gel is required to create an adequate viewing space in most cases. Additional gel should be injected as needed. Gel immersion endoscopy is also helpful for identification of a variety of gastrointestinal bleeding sources, including variceal

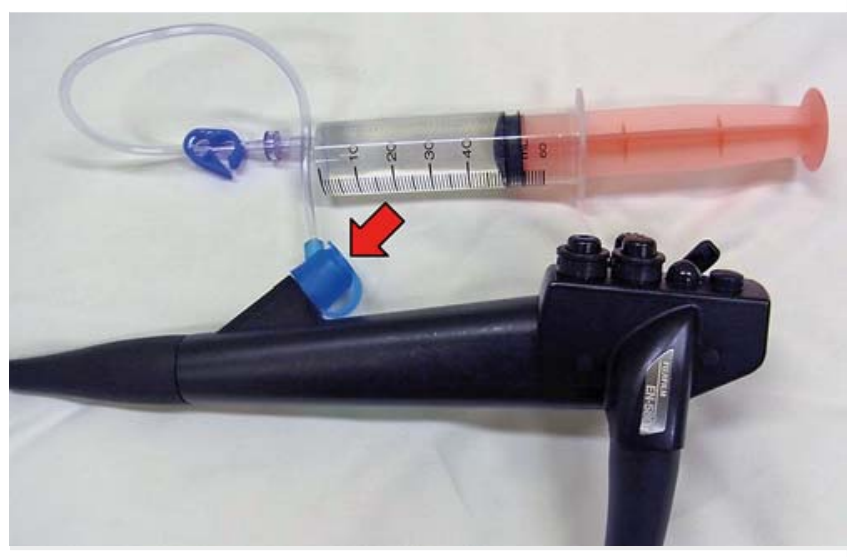

- Fig. 2 A large accessory channel with BioShield irrigator (US Endoscopy (STERIS), Ohio, United States) enables additional injection of gel with a therapeutic device in situ within the accessory channel.

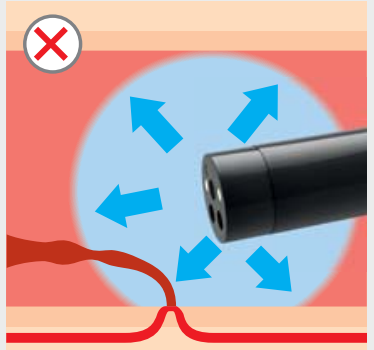

Without a distal attachment

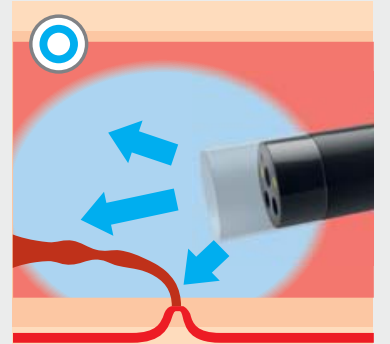

With a distal attachment
Fig. 3 The use of a distal attachment makes it easier to concentrate the gel in front of the scope.

bleeding. This technique facilitates clip hemostasis and injection therapy, but cauterization using mono-polar hemostatic forceps is less effective with this version of the gel "OS-1 jelly," 


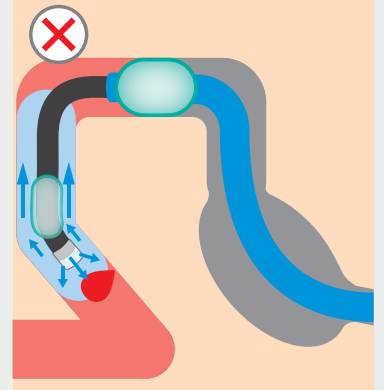

Endoscope-balloon deflated

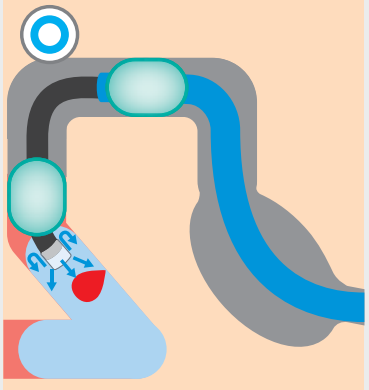

Endoscope-balloon inflated
- Fig.4 Insufflation of the endoscope-balloon can stop a backflow of the gel, making it easier to push away blood, clots and residue.
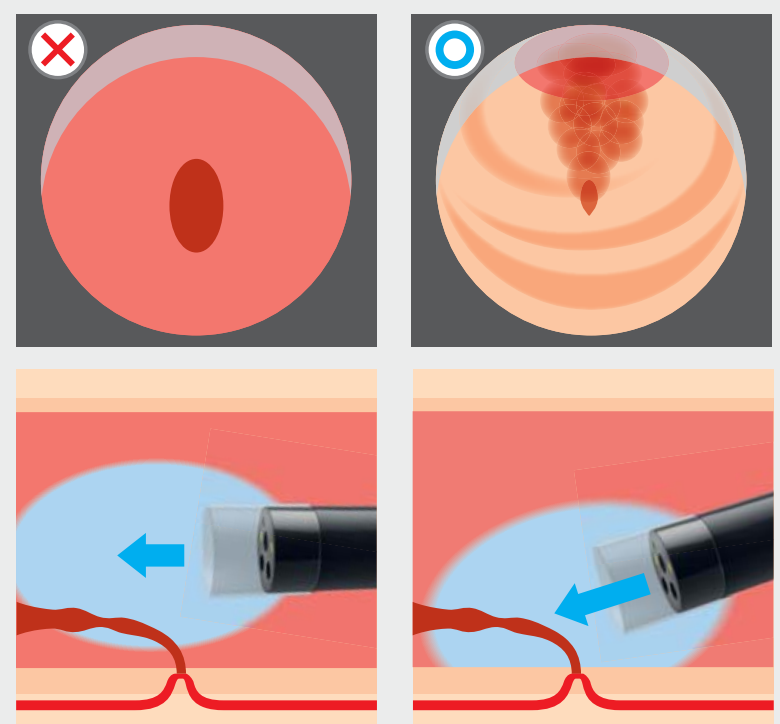

- Fig. 5 The gel should be injected near the bowel wall, below the blood clots to clarify the bleeding point, especially in the stomach, because the bleeding originates from vessels on the wall. Injection in the center of lumen obscures the visual field.

due to dissipation of electric current due to its high electrolyte content. We developed a dedicated electrolyte-free gel "VISCOCLEAR" (Otsuka Pharmaceutical Factory, Tokushima, Japan) for gel immersion endoscopy which was launched in the Japanese market in October 2020.

Procedure-related AEs occurred in four of 265 procedures. However, considering that most procedures were performed for gastrointestinal bleeding in emergency situations, the occurrence rate is not very high. In the two patients with postprocedure abdominal pain, the rapid movement of the infused gel and blood clots or contrast medium by accelerated peristalsis may have caused post-procedure abdominal pain. In the patient with weight gain, not only $400 \mathrm{~mL}$ of gel, but also an unrecorded amount of water infused during the procedure, $1500 \mathrm{~mL}$ of extracellular fluid and 2 units of packed red blood cells transfused before and after the procedure also contributed to the weight gain. In the patient with a spontaneous esophageal rupture, the procedure caused extension of mediastinitis. Because the esophageal rupture was not recognized before performing endoscopy, the extension of mediastinitis occurred due to gas insufflation and water infusion even without gel immersion endoscopy. Although endoscopy in a perforated gastrointestinal tract should be avoided, gel immersion endoscopy facilitates maintaining the luminal pressure lower than gas insufflation and water infusion/immersion. This benefit of gel immersion endoscopy may be useful in a variety of situations where low internal pressure endoscopy is desirable, such as postoperative anastomotic bleeding [1,2] and endoscopic detorsion of a sigmoid volvulus [9].

This study was single center and retrospective. Because the amount of gel and water injected into the lumen were not recorded in many procedures, precise evaluation of the effect on patients is difficult. Further studies are necessary to confirm these preliminary results.

\section{Conclusion}

In conclusion, gel immersion endoscopy is effective and safe for securing the visual field in various locations in the gastrointestinal tract. We recommend using gel immersion endoscopy for refractory gastrointestinal bleeding or in patients with poor preparation.

\section{Competing interests}

Dr. Yano holds a patent as one of the inventors of the dedicated gel for this method. Drs. Yano, Miura, and Sakamoto belong to the Department of Endoscopic Research and International Education (Funded by FUJIFILM Medical and FUJIFILM) and have received honoraria and grants from these corporations. Dr. Yamamoto belongs to the Department of Endoscopic Research and International Education (Funded by FUJIFILM Medical and FUJIFILM) and had a consultant relationship with FUJIFILM Corporation and has received honoraria and grants from these corporations.

References

[1] Frossard JL, Gervaz P, Huber O. Water-immersion sigmoidoscopy to treat acute $\mathrm{Gl}$ bleeding in the perioperative period after surgical colorectal anastomosis. Gastrointest Endosc 2010; 71: 167-170

[2] Gor N, Patil A. Endoscopic management of postoperative ileocolonic anastomotic bleeding by using water submersion. Gastrointest Endosc 2011; 74: 721-722

[3] Yamamoto $\mathrm{H}$, Koiwai $\mathrm{H}$, Sekine $\mathrm{Y}$ et al. Colonoscopy in flowing water for lower $\mathrm{Gl}$ bleeding: a reliable method for confirmation of bleeding points for endoscopic treatment. Gastrointest Endosc 2000; 52: 678681

[4] Yano T, Nemoto D, Ono K et al. Gel immersion endoscopy: a novel method to secure the visual field during endoscopy in bleeding patients (with videos). Gastrointest Endosc 2016; 83: 809-811

[5] Miura Y, Yano T, Takezawa T et al. Gel immersion endoscopy simplifies hemostasis during endoscopic submucosal dissection using the pocket-creation method. Endoscopy 2018; 50: E294-e295 
[6] Akasaka T, Takeuchi Y, Ishida $\mathrm{H}$ et al. A novel gel immersion technique using a bipolar needle-knife in endoscopic submucosal dissection for superficial gastrointestinal neoplasms. Ann Gastroenterol 2018; 31: 247

[7] Yamamoto K, Shiratori Y, Ikeya T. Utility of the gel immersion method for treating massive colonic diverticular bleeding. Clin Endos 2020: doi:10.5946/ce.2020.081
[8] Cotton PB, Eisen GM, Aabakken L et al. A lexicon for endoscopic adverse events: report of an ASGE workshop. Gastrointest Endosc 2010; 71: 446-454

[9] Sugimoto S, Mizukami T, Ito T et al. Endoscopic detorsion for sigmoid volvulus using unsedated water-immersion colonoscopy. Endoscopy 2013; 45: E263-E264 